\title{
SOLID CONTROLLABILITY IN FLUID DYNAMICS
}

\author{
ANDREY A. AGRACHEV ${ }^{1}$ AND ANDREY V. SARYCHEV ${ }^{2}$
}

\begin{abstract}
We survey results of recent activity towards studying controllability and accessibility issues for equations of dynamics of incompressible fluids controlled by low-dimensional or, degenerate, forcing. New results concerning controllability of Navier-Stokes/Euler systems on two-dimensional sphere and on a generic two-dimensional domain are represented.
\end{abstract}

Keywords: incompressible fluid, 2D Euler system, 2D Navier-Stokes system, controllability

AMS Subject Classification: 35Q30, 93C20, 93B05, 93B29

\section{INTRODUCTION}

We survey results of recent activity aimed at studying controllability and accessibility properties of Navier-Stokes systems (NS systems) controlled by low-dimensional (degenerate) forcing. This choice of control is characteristic feature of our problem setting. The corresponding equations are

$$
\begin{array}{r}
\partial u / \partial t+\nabla_{u} u+\operatorname{grad} p=\nu \Delta u+F(t, x), \\
\operatorname{div} u=0 .
\end{array}
$$

The words "degenerate forcing" mean that $F(t, x)$ can be represented as:

$$
F(t, x)=\sum_{k \in \mathcal{K}^{1}} v_{k}(t) F^{k}(x), \mathcal{K}^{1} \text { is finite. }
$$

The word "controlled" means that the functions $v_{k}(t), t \in[0, T]$ entering the forcing can be chosen freely among measurable essentially bounded functions. In fact any functional space, which is dense in $L_{1}[0, T]$ would fit.

The domains treated here include 2-dimensional (compact) Riemannian manifolds $M$ homeomorphic to either sphere, torus or disc. The latter case includes a rectangle and 2D simply connected domain $M$ with analytic boundary $\partial M$. We impose so called Lions boundary condition, whenever boundary is nonempty.

Our approach stems from geometric control theory, which is essentially based on differential geometry and Lie theory; geometric control approach proved its effectiveness in studying controlled dynamics in finite dimensions. We would like to report on some ideas of how such methods can be extended onto the area of infinite-dimensional dynamics and of controlled PDE. Extensions of the geometric control theory onto infinite-dimensional case are 
almost unknown. Classical Lie techniques are not well adapted for infinitedimensional case, and several analytic problems are encountered.

In this contribution we concentrate almost exclusively on geometric and Lie algebraic ideas of the accomplished work. For details on analytic part we refer interested readers to the publications [7, 6, 25, 23, 24, 28, 29].

Applications of geometric theory to the study of controllability of finitedimensional systems is well established subject, although many problems still remain unsolved. Starting point of the activity aimed at controlling NS systems by degenerate forcing was study $([13,4,6,27])$ of accessibility and controllability of their finite-dimensional Galerkin approximations on $\mathbb{T}^{2}$ and $\mathbb{T}^{2}$ (periodic boundary conditions). This question being settled, one should note, that controllability of finite-dimensional Galerkin approximations of NS systems on many other domains remains an open problem; answers for generic analytic 2D domains follow from results of Section 9.

Study of infinite-dimensional case started in $[5,6,7]$, where we dealt with $2 \mathrm{D}$ NS/Euler system on $2 \mathrm{D}$ torus $\mathbb{T}^{2}$. In those publications notions of solid controllability in projections and of approximate controllability have been introduced and sufficient criteria for them have been established.

To arrive to such criteria the technique of so-called Lie extensions in infinite dimensions has been suggested. In the context of our problem this technique can be loosely interpreted as designing the propagation to higher modes of the energy pumped by controlled forcing into lower modes.

The control functions involved are fast-oscillating and analytic part of the study consists of establishing continuity properties of solutions of NS systems with respect to so called relaxation metric of forcing. The latter metric is weaker than the classical metrics and is adapted for dealing with fast oscillating functions.

An extension of the above mentioned techniques techniques onto the case of NS system, subject to Lions boundary conditions on a rectangle, has been accomplished by S.Rodrigues ([23]). In the course of this study both geometric and analytic part needed to be adjusted: Lie extensions turn more intricate and continuity properties need to be reproved. These results are surveyed in Section 8.

A new approach is suggested for establishing controllability on a generic Riemannian surface (Section 9).

Finally study of Lie algebraic properties of spherical harmonics results in controllability criterion for NS/Euler system on 2D sphere (Section 10).

The results appearing in Sections ??-10 have not been previously published.

An interesting extension of the above described methods onto the case of NS system on 3D torus has been accomplished by A.Shirikyan in [28, 29]. The geometric part of his study coincides essentially with the one of [6] and of [27], but additional analytic difficulties in the 3D case are numerous. We do not survey these results for the sake of remaining more geometric in spirit and avoiding new notation. Interested readers should consult [28, 29]. 
There was an extensive study of controllability of the Navier-Stokes and Euler equations in particular by means of boundary control. There are various results on exact local controllability of $2 \mathrm{D}$ and 3D Navier-Stokes equations obtained by A.Fursikov, O.Imanuilov, global exact controllability for 2D Euler equation obtained by J.-M. Coron, global exact controllability for 2D Navier-Stokes equation by A.V. Fursikov and J.-M. Coron. The readers may turn to the book [14] and to the surveys [15] and [11] for further references.

The authors are grateful to S.Rodrigues for useful comments and help during preparation of this contribution.

\section{2D NS/Euler SYSTEM CONTROLled BY DEGENERATE ForCing.}

DEFINITIONS AND PROBLEM SETTING

2.1. NS/Euler system on 2D Riemannian manifold. Representation of NS/Euler system in the form (1)-(2) requires interpretation, whenever one considers the system on 2D domain $M$ with arbitrary Riemannian metric. There is a general way of representing NS/Euler systems on any $n$ dimensional Riemannian manifold (see e.g. [10]), but we prefer to remain in 2 dimensions and to advance with some elementary vector analysis in the 2D Riemannian case.

We consider smooth (or analytic) 2-dimensional Riemannian manifold $M$ (with or without boundary), endowed with Riemannian metric $(\cdot, \cdot)$ and with area 2 -form $\sigma$. All functions, vector fields, forms, we deal with, will be smooth.

Any vector field $y$ on $M$ can be paired with two differential 1-forms defined as:

$$
\begin{array}{r}
y \mapsto y^{b}:\left\langle y^{b}, \xi\right\rangle=(y, \xi) \\
y \mapsto y^{\sharp}:\left\langle y^{\sharp}, \xi\right\rangle=\sigma(y, \xi),
\end{array}
$$

for each vector field $\xi$. Obviously $\left\langle y^{\sharp}, y\right\rangle=\sigma(y, y)=0$.

Note that for any 1-form $\lambda$ there holds:

$$
\lambda \wedge y^{\sharp}=\langle\lambda, y\rangle \sigma .
$$

To prove (4) it suffices to compare the values of 2-forms $\lambda \wedge y^{\sharp}$ and $\langle\lambda, y\rangle \sigma$ on any pair of linearly independent vectors. Evidently (4) is valid if $y$ (and $y^{\sharp}$ ) vanishes. If $y \neq 0$, we take a pair $y, z$, which is linearly independent. Then

$$
\left(\lambda \wedge y^{\sharp}\right)(y, z)=\left|\begin{array}{cc}
\langle\lambda, y\rangle & \left\langle y^{\sharp}, y\right\rangle \\
\langle\lambda, z\rangle & \left\langle y^{\sharp}, z\right\rangle
\end{array}\right|=\langle\lambda, y\rangle \sigma(y, z) .
$$

Now we define the vorticity curl and the divergence div of a vector field via the differentials $d y^{b}, d y^{\sharp}$. These latter are 2-forms; we put

$$
d y^{b}=(\operatorname{curl} y) \sigma, d y^{\sharp}=(\operatorname{div} y) \sigma,
$$

or by abuse of notation:

$$
(\operatorname{curl} y)=d y^{b} / \sigma,(\operatorname{div} y)=d y^{\sharp} / \sigma .
$$


The gradient $\operatorname{grad} \varphi$ of a function $\varphi$ is the vector field paired with $d \varphi$ metrically: $(\operatorname{grad} \varphi)^{b}=d \varphi$.

As in the Euclidean case the vorticity of gradient vector field vanishes:

$$
\operatorname{curl}(\operatorname{grad} \varphi)=d(\operatorname{grad} \varphi)^{b} / \sigma=d(d \varphi) / \sigma=0 .
$$

While in 3D case operator curl transforms vector fields into vector fields, in the $2 \mathrm{D}$ case it transforms vector fields into scalar functions (actually in a component of vector field directed along additional third dimension). We will define the vorticity operator curl on functions. The result of its action on a function $\phi$ is a vector field $\operatorname{curl} \phi$, which satisfies the relation:

$$
\langle\lambda, \operatorname{curl} \phi\rangle \sigma=(d \phi \wedge \lambda),
$$

for each 1-form $\lambda$. By virtue of (4) and due to the nondegeneracy of paring $y \mapsto y^{\sharp}$ we conclude:

$$
(\operatorname{curl} \phi)^{\sharp}=-d \phi .
$$

As in the Euclidean case the divergence of vorticity of a function vanishes:

$$
\operatorname{div}(\operatorname{curl} \phi)=d(\operatorname{curl} \phi)^{\sharp} / \sigma=-d(d \phi) / \sigma=0 .
$$

Coming back to the equation (2) we note that the condition div $u=0$ can be written down as

$$
d u^{\sharp}=0 .
$$

If $M$ is simply connected we conclude that $u^{\sharp}$ must be a differential: $u^{\sharp}=$ $-d \psi$, where $\psi$ is so called stream function. By virtue of (6)

$$
\operatorname{curl} \psi=u \text {. }
$$

For non simply connected domains we impose a condition which guarantees the exactness; in the next subsection we comment on it.

Given the symplectic structure on $M$ defined by $\sigma$ and $(\cdot, \cdot)$ we see that $u$ is Hamiltonian vector field corresponding to the Hamiltonian $-\psi: u=-\vec{\psi}$.

The nonlinear term $\nabla_{u} u$ in the right-hand side of (1) corresponds to covariant derivative of the Riemannian (metric torsion-free) connection on $M$.

Finally we define the Laplace-Beltrami operator $\Delta$ as

$$
\Delta=\operatorname{curl}^{2} .
$$

In Hodge theory (see [10]) this operator transforms $p$-forms into $p$-forms; in our notation $\Delta$ transforms vector fields into vector fields and functions into functions.

2.2. Helmholtz form of $\mathbf{2 D}$ NS sytem. To arrive to the Helmholtz form of the NS system (1)-(2), we apply the operator curl to both parts of (1). As a result we get for the vorticity $\operatorname{curl} u=w$ the equation

$$
\partial w / \partial t+\operatorname{curl}\left(\nabla_{u} u\right)=\nu \Delta w+f(t, x),
$$

where $f(t, x)=\operatorname{curl} F(t, x)$. 
One should note that the vorticity of $\operatorname{grad} p$ vanishes and that the operator curl commutes with $\Delta=\operatorname{curl}^{2}$.

To calculate $\operatorname{curl}\left(\nabla_{u} u\right)$ according to the formula (5)we first compute the 1 -form $\left(\nabla_{u} u\right)^{b}$, adapting the argument of [10, §IV.1.D].

Let $y$ be a vector field which commutes with $u$ : the Lie-Poisson bracket $[u, y]=0$. Then

$$
\left\langle\left(\nabla_{u} u\right)^{b}, y\right\rangle=\left(\nabla_{u} u, y\right)=L_{u}(u, y)-\left(u, \nabla_{u} y\right) .
$$

(Here and below $L_{u}$ denotes Lie derivative. Note that for the covariant derivative of metric connection there holds: $L_{u}(u, y)=\left(\nabla_{u} u, y\right)+\left(u, \nabla_{u} y\right)$.) Since the connection is torsion-free and $[u, y]=0$, then $\nabla_{u} y-\nabla_{y} u=0$, and the right-hand side of (10) can be represented as

$$
L_{u}\left\langle u^{b}, y\right\rangle-\left(u, \nabla_{y} u\right)=L_{u}\left\langle u^{b}, y\right\rangle-\frac{1}{2}\langle d(u, u), y\rangle .
$$

Besides $L_{u}\left\langle u^{b}, y\right\rangle=\left\langle L_{u} u^{b}, y\right\rangle$, as long as $L_{u} y=[u, y]=0$, and we obtain

$$
\left\langle\left(\nabla_{u} u\right)^{b}, y\right\rangle=\left\langle L_{u} u^{b}, y\right\rangle-\frac{1}{2}\langle d(u, u), y\rangle \text {. }
$$

As far as one can find vector field $y$, which commutes with $u$ and has any prescribed value at a given point, we conclude: $\left(\nabla_{u} u\right)^{b}=L_{u} u^{b}-\frac{1}{2} d(u, u)$.

Using the definition of curl (5) we get

$$
\operatorname{curl}\left(\nabla_{u} u\right)=d\left(\left(\nabla_{u} u\right)^{b}\right) / \sigma=d L_{u} u^{b} / \sigma=L_{u} d u^{b} / \sigma=L_{u}(w \sigma) / \sigma=L_{u} w .
$$

Hence $\operatorname{curl}\left(\nabla_{u} u\right)=L_{u} w$.

For $u$ being Hamiltonian vector field with the Hamiltonian $-\psi$ there holds $\nabla_{u} w=-\{\psi, w\}$, where $\{\cdot, \cdot\}$ is Poisson bracket of functions.

The Helmholtz form of the Navier-Stokes equation (cf. [10]) reads

$$
\frac{\partial w}{\partial t}-\{\psi, w\}-\nu \Delta w=f(t, x)
$$

Note that $w=\operatorname{curl} u=\operatorname{curl}^{2} \psi=\Delta \psi$.

The Lions condition written in terms of the vorticity $w$ and the stream function $\psi$ reads:

$$
\left.\psi\right|_{\partial M}=\left.w\right|_{\partial M}=0 .
$$

If the boundary $\partial M$ of $M$ is smooth, then the Hamiltonian vector field $u=-\vec{\psi}$ is tangent to $\partial M$.

Given the vorticity $w$ and the boundary conditions (11), one can recover in a unique way the velocity field $u$, which corresponds to an exact 1 -form $u^{\sharp}$. The corresponding formula is $u=\operatorname{curl} \psi$, where $\psi$ is the unique solution of the Dirichlet problem $\Delta \psi=w$ under boundary condition (11). Indeed such $u$ is divergence free and its vorticity equals $w$ by the definition of $\Delta$.

The NS system can be written as

$$
\frac{\partial w}{\partial t}-\left\{\Delta^{-1} w, w\right\}-\nu \Delta w=f(t, x)
$$


This last equation looks universal: in fact its dependence on the domain is encoded in the properties of the Laplacian $\Delta$ on this domain. It is well explained in $[8,10]$ that the Euler equation for fluid motion is infinitedimensional analogy of Euler equation for rotation of (multidimensional) rigid body, and the Laplacian in (12) plays role of tensor of inertia of rotating rigid body.

2.2.1. Stream function on flat torus. Let us consider the flat torus $\mathbb{T}^{2}$, endowed with standard Riemannian metric and with area form $\sigma$, both inherited from covering of $\mathbb{T}^{2}$ by the Euclidean plane. Let $\varphi_{1}, \varphi_{2}$ be the 'euclidean' coordinates on $\mathbb{T}^{2}$. We proceed in the space of velocities $u$ with vanishing space average: $\int_{\mathbb{T}^{2}} u d \sigma=0$ (due to flatness we may think that all the velocities belong to the same linear space).

To establish exactness of the closed 1-form $u^{\sharp}$ (involved in (8)) it suffices to prove that its integral along a generator of a torus vanishes. By Stokes theorem the integrals of the closed form $u^{\sharp}$ along any two homologous paths have the same value.

Taking $u=\left(u_{1}, u_{2}\right)$ we get $u^{\sharp}=-u_{2} d \varphi_{1}+u_{1} d \varphi_{2}$. Integrating $u^{\sharp}$ along a loop $\Gamma: \varphi_{1}=\alpha$, we obtain the value of the integral $\int_{\Gamma} u^{\sharp}=\int_{0}^{2 \pi} u_{1} d \varphi_{2}=$ $c(\alpha)$, which by the aforesaid is constant: $c(\alpha) \equiv c$. Integrating it with respect to $\varphi_{1}$ we conclude $2 \pi c=\int_{0}^{2 \pi} u_{1} d \varphi_{2} d \varphi_{1}=\int_{\mathbb{T}^{2}} u_{1} d \sigma=0$. Hence $\int_{\Gamma} u^{\sharp}=c=0$. The same holds for the loops $\Gamma^{\prime}: \varphi_{2}=$ const.

2.3. Controllability: definitions. In what follows we reason in terms of so called modes which are the eigenfunctions $\phi^{k}(x)$ of the Laplace-Beltrami operator $\Delta$ defined in the space of vorticities $w: \Delta \phi^{k}(x)=\lambda_{k} \phi^{k}(x)$.

Representing $w, f$ in (12) as a series $w(t, x)=\sum_{k} q_{k}(t) \phi^{k}(x), f(t, x)=$ $\sum_{k} v_{k}(t) \phi^{k}(x)$ with respect to the basis of eigenfunctions one can write the NS system as an infinite system of ODE on the coefficients $q_{k}(t)$. Assume

$$
\left\{\phi^{i}(x), \phi^{j}(x)\right\}=\sum_{k} C_{k}^{i j} \phi^{k}(x) .
$$

Then the equation (12) can be written down 'in coordinate form' as

$$
\dot{q}_{k}-\sum_{i, j} C_{k}^{i j} \lambda_{i}^{-1} q_{i} q_{j}-\nu \lambda_{k} q_{k}=v_{k}(t) .
$$

Typically we will consider the controlled forcing, which is applied to few modes $\phi^{k}(x), k \in \mathcal{K}^{1}, \mathcal{K}^{1}$ - finite. Then in the system (13) the controls enter only the equations indexed by $k \in \mathcal{K}^{1}$, while for $k \notin \mathcal{K}^{1}, v_{k}=0$.

Introduce another finite set $\mathcal{K}^{o}$ of observed modes. We will always assume $\mathcal{K}^{o} \supset \mathcal{K}^{1}$. We identify the space of observed modes with $\mathbb{R}^{N}$ and denote by $\Pi^{o}$ the operator of projection of solutions onto the space of observed modes $\operatorname{span}\left\{\phi_{k} \mid k \in \mathcal{K}^{o}\right\}$. The coordinates corresponding to the observed modes are reunited in observed component $q^{\circ}$.

Galerkin $\mathcal{K}^{\circ}$-approximation of the $2 \mathrm{D}$ NS/Euler system is the equation (ODE) for $q^{o}(t)$, obtained by projecting the 2D NS system onto the space 
of observed modes and putting all the components $q_{k}(t), k \notin \mathcal{K}^{o}$ zero. The resulting equation is

$$
\frac{\partial q^{o}}{\partial t}-\Pi^{o}\left\{\Delta^{-1} q^{o}, q^{o}\right\}-\nu \Delta q^{o}=f(t, x) .
$$

As far as $\mathcal{K}^{o} \supset \mathcal{K}^{1}$, i.e. controlled forcing $f$ only affects part of observed modes, then $\Pi^{o} f(t, x)=f(t, x)$.

In coordinate form passing to Galerkin approximation means omitting the equations (13) for variables $q_{k}$ with $k \notin \mathcal{K}^{o}$ and taking these $q_{k}$ equal 0 in the resting equations.

We say that a control $f(t, x)$ steers the system (12) (or (14)) from $\tilde{\varphi}$ to $\hat{\varphi}$ in time $T$, if for equation (12) forced by $f$ the solution with the initial condition $\tilde{\varphi}$ at $t=0$ takes 'value' $\hat{\varphi}$ at $t=T$.

The first notion of controllability under study is controllability of Galerkin approximation.

Definition 2.1 (controllability of Galerkin approximation). Galerkin $\mathcal{K}^{{ }^{-}}$ approximation of $2 D \mathrm{NS} /$ Euler systems is time-T globally controllable if for any two points $\tilde{q}, \hat{q}$ in $\mathbb{R}^{N}$, there exists a control which steers in time $T$ this Galerkin approximation from $\tilde{q}$ to $\hat{q}$.

This is purely finite-dimensional notion. The next notion regards finitedimensional component of solutions, but takes into account complete infinitedimensional dynamics. Let us introduce some terminology.

Definition 2.2 (attainable sets of NS systems). Attainable set $\mathcal{A}_{\tilde{\varphi}}$ of the $N S / E u l e r$ system (12) is the set of points in $H^{2}(M)$ attained from $\tilde{\varphi}$ by means of essentially bounded measurable controls in any positive time. For each $T>0$ time- $T$ (time- $\leq T)$ attainable set $\mathcal{A}_{\tilde{\varphi}}^{T}\left(\mathcal{A}_{\tilde{\varphi}}^{\leq T}\right)$ of the NS/Euler system is the set of points attained from $\tilde{\varphi}$ by means of essentially bounded measurable controls in time $T$ (in time $\leq T$ ). Attainable set $\mathcal{A}_{\tilde{\varphi}}=\bigcup_{T} \mathcal{A}_{\tilde{\varphi}}^{T}$.

Definition 2.3. The NS/Euler system is time-T globally controllable in projection onto $\mathcal{L}$ if for each $\tilde{\varphi}$ the image $\Pi^{\mathcal{L}}\left(\mathcal{A}_{\tilde{\varphi}}^{T}\right)$ coincides with $\mathcal{L}$.

Definition 2.4. The NS/Euler system is time-T $L_{2}$-approximately controllable if $\mathcal{A}_{\tilde{\varphi}}^{T}$ is $L_{2}$-dense in $H^{2}$.

Let us introduce the notion of accessibility in projection.

Definition 2.5. (accessibility in finite-dimensional projection) Let $\mathcal{L}$ be a finite-dimensional subspace of $H_{2}(M)$ and $\Pi^{\mathcal{L}}$ be $L_{2}$-orthogonal projection of $\mathrm{H}_{2}(\mathrm{M})$ onto $\mathcal{L}$. The $\mathrm{NS} /$ Euler system is time-T accessible in projection on $\mathcal{L}$ if for any $\tilde{\varphi} \in H_{2}(M)$ the image $\Pi^{\mathcal{L}}\left(\mathcal{A}_{\tilde{\varphi}}^{T}\right)$ contains interior points in $\mathcal{L}$.

Now we will introduce notion of solid controllability.

Definition 2.6. Fix initial condition $\tilde{\varphi} \in H_{2}(M)$ for trajectories of the controlled $2 D \mathrm{NS} /$ Euler system. Let $v(\cdot) \in L_{\infty}\left([0, T] ; \mathbb{R}^{r}\right)$ be the controlled forcing and $w_{t}$ be the corresponding trajectory of the NS system. 
If NS/Euler system is considered on an interval $[0, T](T<+\infty)$, then the map $E_{T}: v(\cdot) \mapsto w_{T}$ is called end-point map; the map $\Pi^{o} \circ \mathcal{F} / \mathcal{T}_{T}$ is called end-point component map, the composition $\Pi^{\mathcal{L}} \circ \mathcal{F} / \mathcal{T}_{T}$ is called $\mathcal{L}$-projected end-point map.

Definition 2.7. Let $\Phi: \mathcal{M}^{1} \mapsto \mathcal{M}^{2}$ be a continuous map between two metric spaces, and $S \subseteq \mathcal{M}^{2}$ be any subset. We say that $\Phi$ covers $S$ solidly, if $S \subseteq \Phi\left(\mathcal{M}^{1}\right)$ and this inclusion is stable with respect to $C^{0}$-small perturbations of $\Phi$, i.e. for some $C^{0}$-neighborhood $\Omega$ of $\Phi$ and for each map $\Psi \in \Omega$, there holds: $S \subseteq \Psi\left(\mathcal{M}^{1}\right)$.

Definition 2.8. (solid controllability in finite-dimensional projection) The $2 D \mathrm{NS} /$ Euler system is time-T solidly globally controllable in projection on finite-dimensional subspace $\mathcal{L} \subset H^{2}(M)$, if for any bounded set $S$ in $\mathcal{L}$ there exists a set of controls $B_{S}$ such that $\left(\Pi^{\mathcal{L}} \circ \mathcal{F} / \mathcal{T}_{T}\right)\left(B_{S}\right)$ covers $S$ solidly.

2.4. Problem setting. In this contribution we will discuss the following questions.

- Under what conditions the 2D NS/Euler system is globally controllable in observed component?

- Under what conditions the 2D NS/Euler system is solidly controllable in a finite-dimensional projection?

- Under what conditions the 2D NS/Euler system is accessible in a finite-dimensional projection?

- Under what conditions the 2D NS/Euler system is $L_{2}$-approximately controllable?

As we explained above the geometry of controllability is encoded in spectral properties of the Laplacian $\Delta$ and therefore on the geometry of the domain on which the controlled NS system evolves. Below we provide answers for particular types of domains.

\section{Geometric CONTROL: ACCESSibility And CONTRollability Via LIE BRACKETS}

In this section we collect some results of geometric control theory regarding accessibility and controllability of finite-dimensional real-analytic controlaffine systems of the form

$$
\dot{x}=f^{0}(x)+\sum_{i=1}^{r} f^{i}(x) v_{i}(t), x(0)=x^{0}, v_{i}(t) \in \mathbb{R}, i=1, \ldots, r .
$$

Geometric approach is coordinate-free, so that it is adapted for dealing with dynamics on manifolds, but we will assume that the system (15) is defined on a finite-dimensional linear space $\mathbb{R}^{N}$ in order to maintain parallelism with NS systems, which evolve in Hilbert spaces.

We use standard notation $P_{t}=e^{t f}$ for the flow corresponding to a vector field $f$. 
3.1. Orbits, Lie rank accessibility. Let $v(\cdot) \in L_{\infty}\left([0, T] ; \mathbb{R}^{r}\right)$ be admissible controls and $x(t)$ be corresponding trajectories of the system $\dot{x}=$ $f^{0}(x)+\sum_{i=1}^{r} f^{i}(x) v_{i}(t)$ with initial point $x(0)=x^{0}$. We again introduce the end-point map $\mathcal{E}_{T}: v(\cdot) \mapsto x_{v}(T)$; here $x_{v}(\cdot)$ is the trajectory of (15) corresponding to the control $v(\cdot)$.

For each $T>0$ time- $T$ (time- $\leq T$ ) attainable set $\mathcal{A}_{x^{0}}^{T}$ from $x^{0}$ of the system $(15)$ is the image of the set $L_{\infty}\left([0, T] ; \mathbb{R}^{r}\right)$ under the map $\mathcal{E}_{T}$, or, equivalently, the set of points $x(T)$ attained in time $T$ from $x^{0}$ by means of admissible controls. The time- $\leq T$ attainable set from $x^{0}$ is $\mathcal{A}_{x^{0}}^{\leq T} \bigcup_{t \in[0, T]} \mathcal{A}_{x^{0}}^{t}$. Attainable set from $x^{0}$ of the system (15) is $\mathcal{A}_{x^{0}}=\bigcup_{T>0} \mathcal{A}_{x^{0}}^{T}$.

Important notions of geometric control theory are orbits of control system.

Definition 3.1 (orbits and zero-time orbits of control systems). An orbit of the control system (15) passing through $x^{0}$ is the set of points obtained from $x^{0}$ under the action of (the group of) diffeomorphisms of the form $e^{t_{1} f^{u^{1}}} \circ \cdots \circ$ $e^{t_{N} f^{u^{N}}}$, where $t_{j} \in \mathbb{R}, j=1, \ldots, N$, and $f^{u^{j}}=f^{0}+\sum_{i=1}^{r} f^{i}(x) u_{i}^{j}$ is the righthand side of (15) corresponding to constant control $u^{j}=\left(u_{1}^{j}, \ldots, u_{r}^{j}\right) \in \mathbb{R}^{r}$. Zero-time orbit is the subset of the orbit, resulting from the action of these diffeomorphisms subject to condition $\sum_{j} t_{j}=0$.

If we consider 'symmetrization' of the system (15),

$$
\dot{x}=f^{0}(x) v_{0}+\sum_{i=1}^{r} f^{i}(x) v_{i}(t), x(0)=x^{0}, v_{0} \in \mathbb{R}, v_{i}(t) \in \mathbb{R}, i=1, \ldots, r,
$$

then the orbit of (15) can be interpreted as the attainable set from $x^{0}$ of this symmetrization corresponding to application of piecewise-constant controls.

The famous Nagano theorem relates properties of the orbits and Lie algebraic properties of the system. It claims that the orbit and the zero-time orbit of an analytic system (15) are immersed manifolds of $\mathbb{R}^{N}$, and tangent spaces to these orbits can be calculated via Lie brackets of the vector fields $\left\{f^{0}, \ldots, f^{m}\right\}$.

Definition 3.2 (Lie rank and zero-time Lie rank). Take the Lie algebra Lie $\left\{f^{0}, \ldots, f^{m}\right\}$ generated by $\left\{f^{0}, \ldots, f^{m}\right\}$ and evaluate vector fields from Lie $\left\{f^{0}, \ldots, f^{m}\right\}$ at a point $x$; the dimension of the resulting linear space Lie $_{x}\left\{f^{0}, \ldots, f^{m}\right\}$ is Lie rank of the system $\left\{f^{0}, \ldots, f^{m}\right\}$ at $x$.

Take the Lie ideal generated by span $\left\{f^{1}, \ldots, f^{m}\right\}$ in $\operatorname{Lie}\left\{f^{0}, \ldots, f^{m}\right\}$ and evaluate vector fields from it at $x$; the dimension of the resulting linear space $L i e_{x}^{0}\left\{f^{0}, \ldots, f^{m}\right\}$, is zero-time Lie rank at $x$ of the system $\left\{f^{0}, \ldots, f^{m}\right\}$.

These two Lie ranks either are equal or differ by 1 .

The Nagano theorem claims that in analytic case $\operatorname{Lie}_{x}\left\{f^{0}, \ldots, f^{m}\right\}$ and $\operatorname{Lie}_{x}^{0}\left\{f^{0}, \ldots, f^{m}\right\}$ are tangent spaces at each point $x$ of the orbit and zerotime orbit respectively.

Accessibility properties of analytic control system (15) are determined by its Lie ranks. Recall that the system is called accessible if its attainable set 
$\mathcal{A}_{x^{0}}$ has nonempty interior and is strongly accessible if $\forall T>0$ attainable sets $\mathcal{A}_{x^{0}}^{T}$ have nonempty interior. The following fact holds.

Theorem 3.3 (Jurdjevic-Sussmann $\left(C^{\omega}\right.$ case), Krener $\left(C^{\infty}\right.$ case)). If the Lie rank of the system of vector fields $\left\{f^{0}, \ldots, f^{r}\right\}$ at $x^{0}$ equals $n$, then $\forall T>0$ the interior of the attainable set $\mathcal{A}_{x^{0}}^{\leq T}$ is nonvoid. Besides $\mathcal{A}_{x^{0}}^{\leq T}$ possesses the interior which is dense in it. If zero-time Lie rank at $x^{0}$ equals $n$, then $\forall T>0$ the interior of the attainable set $\mathcal{A}_{x^{0}}^{T}$ is nonvoid and this interior is dense in $\mathcal{A}_{x^{0}}^{T}$.

See $[19,2]$ for the proof.

Let $\mathcal{L}$ be a linear subspace of $\mathbb{R}^{N}$ and $\Pi^{\mathcal{L}}$ be a projection of $\mathbb{R}^{N}$ onto $\mathcal{L}$. A control system (15) is accessible (strongly accessible) from $x$ in projection on $\mathcal{L}$ if the image $\Pi^{\mathcal{L}} \mathcal{A}_{x^{0}}\left(\Pi^{\mathcal{L}} \mathcal{A}_{x^{0}}^{T}\right)$ contains interior points in $\mathcal{L}$ (for each $T>0)$.

One easily derives from the previous theorem a criterion for accessibility in projection.

Theorem 3.4. If $\Pi^{\mathcal{L}}$ maps $\operatorname{Lie}_{x}\left\{f^{0}, \ldots, f^{m}\right\}$ (respectively $\operatorname{Lie}_{x}^{0}\left\{f^{0}, \ldots, f^{m}\right\}$ ) onto $\mathcal{L}$, then control system (15) is accessible (respectively strongly accessible) at $x$ in projection on $\mathcal{L}$.

Proof. The proofs of the two statements are similar; we sketch the proof of the first one. Consider the orbit of the system (15) passing through $x_{0}$. The tangent space to the orbit at each of its points $x$ coincides with $\operatorname{Lie}_{x}\left\{f^{0}, \ldots, f^{m}\right\}$.

By Theorem 3.3 the attainable set of the system possesses relative interior with respect to the orbit. Moreover there are interior points $x_{\text {int }} \in \mathcal{A}_{x^{0}}$ arbitrarily close to $x^{0}$ so that $\Pi^{\mathcal{L}}$ maps $\operatorname{Lie}_{x_{i n t}}\left\{f^{0}, \ldots, f^{m}\right\}$ onto $\mathcal{L}$. Then sufficiently small neighborhoods of $x_{i n t}$ in the orbit are contained in $\mathcal{A}_{x^{0}}$ and are mapped by $\Pi^{\mathcal{L}}$ onto a subset of $\mathcal{L}$ with nonempty interior.

3.2. Lie extensions and controllability. Controllability is stronger and much more delicate property than accessibility. To verify it it does not suffice in general to compute the Lie rank which accounts for all the Lie brackets. Instead one should select 'good Lie brackets' avoiding 'bad Lie brackets' or 'obstructions'.

To have a general idea of what good and bad Lie brackets can be like let us consider the following elementary example.

\section{Example 1.}

$$
\dot{x}_{1}=v, \dot{x}_{2}=x_{1}^{2} .
$$

This is 2-dimensional control-affine system (15) with $f^{0}=x_{1}^{2} \partial / \partial x_{2}, f^{1}=$ $\partial / \partial x_{1}$. The Lie rank of this system equals 2 at each point, the system is accessible but uncontrollable from each point $\hat{x}=\left(\hat{x}_{1}, \hat{x}_{2}\right)$ given the fact that we can not achieve points with $x_{2}<\hat{x}_{2}$. One can prove that the attainable set $\mathcal{A}_{\hat{x}}$ coincides with the half-plane $x_{2}>\hat{x}_{2}$ with added point $\hat{x}$. One sees 
that that it is possible to move freely (bidirectionally) along good vector field $f^{1}$, while along bad Lie bracket $\left[f^{1}\left[f^{1}, f^{0}\right]\right]=2 \partial / \partial x_{2}$ we can move only in one direction.

Good Lie brackets form Lie extension of a control system.

Definition 3.5. The family $\mathcal{F}^{\prime}$ of real analytic vector fields is:

i) an extension of $\mathcal{F}$ if $\mathcal{F}^{\prime} \supset \mathcal{F}$ and the closures of the attainable sets $\mathcal{A}_{\mathcal{F}}(\tilde{x})$ and $\mathcal{A}_{\mathcal{F}^{\prime}}(\tilde{x})$ coincide;

ii) time- $T$ extension of $\mathcal{F}$ if $\mathcal{F}^{\prime} \supset \mathcal{F}$ and $\forall T>0$ the closures of the time- $T$ attainable sets $\mathcal{A}_{\mathcal{F}}^{T}(\tilde{x})$ and $\mathcal{A}_{\mathcal{F}^{\prime}}^{T}(\tilde{x})$ coincide;

iii) fixed-time extension if it is time-T extension $\forall T>0$.

The vector fields from $\mathcal{F}^{\prime} \backslash \mathcal{F}$ are called i) compatible, ii) compatible in time $T$, iii) compatible in a fixed time with $\mathcal{F}$ in the cases i), ii) and iii) correspondingly.

The inclusions $\mathcal{A}_{\mathcal{F}}(\tilde{x}) \subset \mathcal{A}_{\mathcal{F}^{\prime}}(\tilde{x}), \mathcal{A}_{\mathcal{F}}^{T}(\tilde{x}) \subset \mathcal{A}_{\mathcal{F}^{\prime}}^{T}(\tilde{x})$ are obvious. Less obvious is the following Proposition (see [2]).

Proposition 3.6. If an extension $\mathcal{F}^{\prime}$ of an analytic system $\mathcal{F}$ is globally controllable, then $\mathcal{F}^{\prime}$ also is.

Remark 3.1. When talking about time-T extensions one can consider also extensions by time-variant vector fields $X_{t}, t \in[0, T]$. We will say that vector field $X_{t}$ is time- $T$ compatible with $\mathcal{F}$ if it drives the system in time $T$ from $\tilde{x}$ to the closure of $\mathcal{A}_{\mathcal{F}}^{T}(\tilde{x})$.

Our idea is to proceed with a series of extensions of a control system in order to arrive to extended system for which the controllability can be verified and then apply Proposition 3.6.

Obviously the Definition 3.5 is nonconstructive. In what follows we will use three particular types of extensions.

The first natural type is based on possibility of taking topological closure of a set of vector fields, maintaining closures of attainable sets.

Proposition 3.7. (see [19, Ch. 3, §2, Th.5]) Topological (with respect to $C^{\infty}$ convergence on compact sets) closure $\operatorname{cl}(\mathcal{F})$ of $\mathcal{F}$ is Lie extension.

The second method underlies the theory of relaxed (or sliding mode) controls started $[17,16]$, which provided an extension of pioneering contributions by L.C.Young [30] and E.J. McShane [22] onto the field of optimal control theory. To introduce it we consider a family of so-called relaxation seminorms $\|\cdot\|_{s, K}$ of time-variant vector fields $X_{t}, t \in[0, T]$ :

$$
\|X .\|_{s, K}^{\mathrm{rx}}=\max _{t \in[0, T]}\left\|\int_{0}^{t} X_{\tau} d \tau\right\|_{s, K},
$$

with $K$ being a compact in $\mathbb{R}^{N}, s \geq 0$ being an integer and $\left\|X_{\tau}\right\|_{s, K}$ being a $C^{k}$-norm on the compact $K$. The family of relaxation seminorms define relaxation $\left(C^{\infty}\right)$ topology (metrics) in the set of time-variant vector fields. 
Proposition 3.8 (see $[17,2])$. Let a sequence of time-variant vector fields $X_{t}^{j}$ converge to $X_{t}$ in the relaxation metrics and let these vector fields have compact supports. Then the flows of the vector fields $X_{t}^{j}$ converge to the flow of the vector field $X_{t}$.

As a corollary of this result one can prove

Proposition 3.9. For the systems

$\operatorname{co} \mathcal{F}=\left\{\sum_{i=1}^{m} \beta_{i} f_{i}, f_{i} \in \mathcal{F}, \beta_{i} \in C^{\omega}\left(\mathbb{R}^{N}\right), \beta_{i} \geq 0, \sum_{i=1}^{m} \beta_{i} \equiv 1, i=1, \ldots, m\right\}$,

and $\mathcal{F}$ the closures of their time-T attainable sets coincide.

Proof of this result and of its modifications can be found in [2, Chapter 8], [19, Chapter 3],[17, Chapters II,III ].

The next type of extension, we will use, relies upon Lie brackets. It appeared in our earlier work on controllability of Euler equation for rigid body in [3] and was named there reduction of a control-affine system. Here we present a particular version adapted to our problem. Repeated application of this extension settles controllability issue for finite-dimensional Galerkin approximations of NS systems.

Proposition 3.10. Consider control-affine analytic system

$$
\dot{x}=f^{0}(x)+f^{1}(x) \hat{v}_{1}+f^{2}(x) \hat{v}_{2} .
$$

Let

$$
\left[f^{1}, f^{2}\right]=0,\left[f^{1},\left[f^{1}, f^{0}\right]\right]=0 .
$$

Then the system

$$
\dot{x}=f^{0}(x)+f^{1}(x) \tilde{v}_{1}+f^{2}(x) \tilde{v}_{2}+\left[f^{1},\left[f^{2}, f^{0}\right]\right] v_{12},
$$

is fixed-time Lie extension of (17).

Sketch of the proof. Take Lipschitzian functions $v_{1}(t), v_{2}(t),\left(v_{1}(0)=\right.$ $\left.v_{2}(0)=0\right)$ and change in $(17) \hat{v}_{1}, \hat{v}_{2}$ to $\varepsilon^{-1} \dot{v}_{1}(t)+\tilde{v}_{1}$ and $\varepsilon \dot{v}_{2}(t)+\tilde{v}_{2}$ respectively. We arrive to the equation

$$
\dot{x}=f^{0}(x)+f^{1}(x) \tilde{v}_{1}+f^{2}(x) \tilde{v}_{2}+\left(\varepsilon^{-1} f^{1} \dot{v}_{1}(t)+\varepsilon f^{2} \dot{v}_{2}(t)\right) .
$$

Applying the 'reduction formula' from [3] or alternatively 'variation of constants' formula of chronological calculus ([1]) one can represent the flow of (19) as a composition of the flow $\tilde{P}_{t}$ of the equation

$$
\dot{y}=e^{\operatorname{ad} f^{1}(y) \varepsilon^{-1} v_{1}(t)+\operatorname{ad} f^{2} \varepsilon v_{2}(t)} f^{0}(y)+f^{1}(x) \tilde{v}_{1}+f^{2}(x) \tilde{v}_{2} .
$$

and the flow

$$
P_{t}=e^{f^{1} \varepsilon^{-1} v_{1}(t)+f^{2} \varepsilon v_{2}(t)} .
$$

For the validity of this decomposition the equality $\left[f^{1}, f^{2}\right]=0$ is important. 
In (20) $e^{\operatorname{ad}_{f}}$ is exponential of the operator $\operatorname{ad}_{f}: e^{\operatorname{ad}_{f}}=\sum_{j=0}^{\infty}\left(\operatorname{ad}_{f}\right)^{j} / j$ !. The operator ad $f$ is determined by the vector field $f$ and acts on vector fields as: $\operatorname{ad}_{f} g=[f, g],[f, g]$ being the Lie bracket of $f$ and $g$.

By first of the relations (18) operators $\operatorname{ad}_{f^{1}}$ and $\operatorname{ad}_{f^{2}}$ commute and by the second of these relations any iterated Lie bracket of the form $\left(\operatorname{ad}_{f^{i_{1}}}\right) \circ \cdots \circ$ $\left(\operatorname{ad}_{f^{i m}}\right) f^{0}$, with $i_{j}=1,2$, vanishes whenever it contains $\operatorname{ad}_{f^{1}}$ at least twice.

Taking the expansion of the operator exponential in (20) and using these facts we get ${ }^{1}$

$$
\begin{array}{r}
\dot{y}=f^{0}(y)+f^{1}(x) \tilde{v}_{1}+f^{2}(x) \tilde{v}_{2}+ \\
+\varepsilon^{-1}\left[f^{1}, f^{0}\right](x) v_{1}(t)+\left[f^{1},\left[f^{2}, f^{0}\right]\right](x) v_{1}(t) v_{2}(t)+O(\varepsilon),
\end{array}
$$

To obtain the flow of (19) we need to compose the flow of (22) with the flow (21). For any fixed $T$ one can get $P_{T}=I d$ by choosing $v_{1}(\cdot), v_{2}(\cdot)$ such that $v_{1}(T)=v_{2}(T)=0$.

From now on we deal with the fixed $T$ and the flow of the equation (22).

Let us change in $(22) v_{j}(t)$ to $v_{j}(t)=2^{1 / 2} \sin \left(t / \varepsilon^{2}\right) \bar{v}_{j}(t)$, with $\bar{v}_{j}(t)$ being functions of bounded variation $j=1,2$. The relaxation seminorms of the time-variant vector field $\varepsilon^{-1}\left[f^{1}, f^{0}\right](x) 2^{1 / 2} \sin \left(t / \varepsilon^{2}\right) \bar{v}_{1}(t)$ in the right-hand side of $(22)$ are $O(\varepsilon)$ as $\varepsilon \rightarrow+0$. Also in the right-hand side of $(22)$

$$
\begin{array}{r}
{\left[f^{1},\left[f^{2}, f^{0}\right]\right](x) 2 \sin ^{2}\left(t / \varepsilon^{2}\right) \bar{v}_{1}(t) \bar{v}_{2}(t)=} \\
=\left[f^{1},\left[f^{2}, f^{0}\right]\right](x) \bar{v}_{1}(t) \bar{v}_{2}(t)-\left[f^{1},\left[f^{2}, f^{0}\right]\right](x) \cos \left(2 t / \varepsilon^{2}\right) \bar{v}_{1}(t) \bar{v}_{2}(t) .
\end{array}
$$

The relaxation seminorms of the addend $\left[f^{1},\left[f^{2}, f^{0}\right]\right](x) \cos \left(2 t / \varepsilon^{2}\right) \bar{v}_{1}(t) \bar{v}_{2}(t)$ are $O\left(\varepsilon^{2}\right)$ as $\varepsilon \rightarrow+0$.

Hence the right-hand sides of the equation (22) with the controls $v_{j}(t)=$ $2^{1 / 2} \sin \left(t / \varepsilon^{2}\right) \bar{v}_{j}(t), j=1,2$, converge in relaxation metric to the vector field

$$
f^{0}(y)+f^{1}(x) \tilde{v}_{1}+f^{2}(x) \tilde{v}_{2}+\left[f^{1},\left[f^{2}, f^{0}\right]\right](x) \bar{v}_{1}(t) \bar{v}_{2}(t)
$$

as $\varepsilon \rightarrow 0$. We can consider the product $\bar{v}_{1}(t) \bar{v}_{2}(t)$ as a new control $v_{12}$ and invoke the Proposition 3.8.

\section{BRACKET COMPUTATION IN Finite AND INFINITE DimENSIONS; CONTROLLING ALONG "PRINCIPAL AXES"}

In this section we adjust the statement of the Proposition 3.8 for studying controllability of the systems (12) and (14).

From viewpoint of geometric control the Galerkin approximation (14) of NS/Euler system is particular type of control-affine system (15). Its state space is finite-dimensional and is generated by a finite number of eigenfunctions of the Laplace-Beltrami operator $\Delta$ or modes.

\footnotetext{
${ }^{1}$ The time-variant vector field abbreviated by $O(\varepsilon)$ in $(22)$ equals $\varepsilon \phi\left(\operatorname{sad}_{v_{2}(t) f^{2}}\right) \operatorname{ad}_{v_{2}(t) f^{2}} f^{0}+\varepsilon^{2} \operatorname{ad}_{v_{2}(t) f^{2}}^{2} \varphi\left(\varepsilon \operatorname{ad}_{v_{2}(t) f^{2}}\right)\left[f^{1}, f^{0}\right]$, where $\phi(z)=z^{-1}\left(e^{z}-1\right), \varphi(z)=z^{-2}\left(e^{z}-1-z\right)$.
} 
The dynamics of this control system is determined by quadratic drift vector field

$$
f_{o}^{0}=\Pi^{o}\left\{\Delta^{-1} q^{o}, q^{o}\right\}+\nu \Delta q^{o}
$$

and by controlled forcing $\sum_{i=1}^{r} f^{i}(x) u_{i}$, where $f^{i}$ are constant $\left(q^{o}\right.$-independent) controlled vector fields.

Start with computation of the particular Lie brackets, which are involved in the formulation of the Proposition 3.8. For two constant vector fields $f^{1}, f^{2}$ there holds

$$
\begin{array}{r}
{\left[f^{i}, f_{o}^{0}\right]=\Pi^{o}\left(\left\{f^{i}, \Delta^{-1} w\right\}+\left\{w, \Delta^{-1} f^{i}\right\}\right)+\nu \Delta f^{i}, i=1,2 ;} \\
{\left[f^{1},\left[f^{2}, f_{o}^{0}\right]\right]=\Pi^{o}\left(\left\{f^{2}, \Delta^{-1} f^{1}\right\}+\left\{f^{1}, \Delta^{-1} f^{2}\right\}\right),} \\
{\left[f^{1},\left[f^{1}, f_{o}^{0}\right]\right]=2 \Pi^{o}\left\{f^{1}, \Delta^{-1} f^{1}\right\} .}
\end{array}
$$

This computation is finite-dimensional but the same holds true if one considers constant vector fields acting in infinite-dimensional Hilbert space. Taking 'drift' vector field of the equation (12) in infinite dimensions

$$
f^{0}=\left\{\Delta^{-1} q, q\right\}+\nu \Delta q .
$$

we conclude with

Lemma 4.1. For two constant vector fields $f^{1}, f^{2}$ there holds

$$
\begin{array}{r}
{\left[f^{i}, f^{0}\right]=\left\{f^{i}, \Delta^{-1} w\right\}+\left\{w, \Delta^{-1} f^{i}\right\}+\nu \Delta f^{i}, i=1,2} \\
{\left[f^{1},\left[f^{1}, f^{0}\right]\right]=2\left\{f^{1}, \Delta^{-1} f^{1}\right\}} \\
\mathcal{B}\left(f^{1}, f^{2}\right)=\left[f^{1},\left[f^{2}, f^{0}\right]\right]=\left\{f^{2}, \Delta^{-1} f^{1}\right\}+\left\{f^{1}, \Delta^{-1} f^{2}\right\} .
\end{array}
$$

Let us see what is needed for the assumptions of the Proposition 3.10 to hold. As long as $f^{1}, f^{2}$ are constant and hence commuting, all we need is

$$
\left[f^{1},\left[f^{1}, f^{0}\right]\right]=\left\{f^{1}, \Delta^{-1} f^{1}\right\}=0 .
$$

In terms of Euler equation for ideal fluid

$$
\frac{\partial w}{\partial t}-\left\{\Delta^{-1} w, w\right\}=0
$$

this means that $f^{1}$ corresponds to its steady motion. In particular eigenfunctions of the Laplace-Beltrami operator $\Delta$ correspond to steady motions and satisfy (24). In what follows these eigenfunctions will be used as controlled directions.

Eigenfunctions of the Laplacian are analogous to principal axes of a (multidimensional) rigid body.

By Proposition 3.10 given two constant controlled vector fields one of which corresponds to a steady motion we can extend our control system by new controlled vector field (23) which is again constant.

Our method consists of iterating this procedure. The algebraic/geometric difficulties, which arise on this way, consist of scrutinizing the newly obtained controlled directions. In particular we want to know whether at each step 
we obtain a proper extension. This will be illustrated in the further Sections which deal with particular 2D domains.

Another (analytic) difficulty arises when we pass from finite-dimensional approximations to controlled PDE. For these latter the sketched above proof of the Proposition 3.10 is not valid anymore (for example one can not speak about flows). We have to reprove statement of the Proposition in each particular situation. The main idea will be still based on using fast oscillating control and relaxation metric. The analytic difficulties are in proving continuity of forcing/trajectory map with respect to such metric. We will provide a brief comment in the next Section; the readers can find details in $[6,7,23,24]$.

\section{Controllability and accessibility of Galerkin APPROXIMATIONS OF NS/EULER SYSTEM ON $\mathbb{T}^{2}$}

Here we survey results on accessibility and controllability of Galerkin approximations of $2 \mathrm{D} \mathrm{NS} /$ Euler system on $\mathbb{T}^{2}$.

5.1. Accessibility of Galerkin approximations. The result of the computation (23) in the periodic case is easy to visualize when the constant controlled vector fields which correspond to the eigenfunctions of Laplacian $\Delta$ on $\mathbb{T}^{2}$, are written as complex exponentials.

For two different complex eigenfunctions $f^{1}=e^{i k \cdot x}, f^{2}=e^{i \ell \cdot x}$ of the Laplacian $\Delta$ on $\mathbb{T}^{2}$, where $x \in \mathbb{R}^{2}, k, \ell \in \mathbb{Z}^{2}$, the Poisson bracket in (23) equals

$$
\mathcal{B}\left(e^{i k \cdot x}, e^{i \ell \cdot x}\right)=(k \wedge \ell)\left(|k|^{-2}-|\ell|^{-2}\right) e^{i(k+\ell) \cdot x},
$$

i.e. again corresponds to an eigenfunction of $\Delta$, provided that $|k| \neq|\ell|, k \wedge$ $\ell \neq 0$. The conclusion is that given two pairs of complex exponentials $e^{ \pm i k \cdot x}, e^{ \pm i \ell \cdot x}$ as controlled vector fields we can extend them by controlled vector fields $e^{i( \pm k \pm \ell) \cdot x}$.

Iterating the computation of the Lie-Poisson brackets (23) and obtaining new directions we end up with (finite or infinite) set of functions, which contains $e^{ \pm i k \cdot x}, e^{ \pm i \ell \cdot x}$ and is invariant with respect to the bilinear operation $\mathcal{B}(\cdot, \cdot)$.

Therefore in the case of $\mathbb{T}^{2}$ starting with controlled vector fields, which correspond to the eigenfunctions $e^{i k \cdot x}, k \in \mathbb{Z}^{2}$, of the Laplacian, the whole computation of Lie extensions 'can be modeled' on the integer lattice $\mathbb{Z}^{2}$ of "mode indices" $k$.

Actually one has to operate with real eigenfunctions of the Laplacian on $\mathbb{T}^{2}$, i.e. with the functions of the form $\cos (k \cdot x), \sin (k \cdot x)$. Also in this case computation of iterated Lie-Poisson brackets (23) can be modelled on $\mathbb{Z}^{2}$ and the addition formulae are similar to those for the complex case.

We formulate now bracket generating criterion. 
Proposition 5.1 (bracket generating property). If

$$
|k| \neq|\ell|,|k \wedge \ell|=1,
$$

then:

i) invariant with respect to $\mathcal{B}$ set of functions, which contains complex functions $e^{ \pm i k \cdot x}, e^{ \pm i \ell \cdot x}$, contains all the eigenfunctions $e^{i m \cdot x}, m \in \mathbb{Z}^{2} \backslash 0$;

ii) invariant with respect to $\mathcal{B}$ set of real functions, which contains

$$
\cos (k \cdot x), \sin (k \cdot x), \cos (\ell \cdot x), \sin (\ell \cdot x),
$$

contains all the eigenfunctions $\cos (m \cdot x), \sin (m \cdot x), m \in \mathbb{Z}^{2} \backslash 0$.

Bracket generating property for Galerkin approximation of 2D NS system under periodic boundary conditions has been established by W.E and J.Mathingly in [13]. The following result from [13] is an immediate consequence of the previous Proposition 5.1 and Theorem 3.3.

Corollary 5.2 (accessibility by means of 4 controls). For any subset $\mathcal{M} \subset$ $\mathbb{Z}^{2}$ there exists a larger set $\mathcal{M}^{\prime} \supseteq \mathcal{M}$ such that Galerkin $\mathcal{M}^{\prime}$-approximation controlled by the forcing

$$
\cos (k \cdot x) v_{k}(t)+\sin (k \cdot x) w_{k}(t)+\cos (\ell \cdot x) v_{\ell}(t)+\sin (\ell \cdot x) w_{\ell}(t),
$$

with $k, \ell$ satisfying (26), is strongly accessible.

Here 4 controls $v_{k}(t), w_{k}(t), v_{\ell}(t), w_{\ell}(t)$ are used for providing strong accessibility, but actually it can be achieved by a smaller number of controls.

Example (accessibility by means of 2 controls) Consider forcing

$$
g v(t)+\bar{g} \bar{v}(t), g=\cos (k \cdot x)+\cos (\ell \cdot x), \bar{g}=\sin (k \cdot x)-\sin (\ell \cdot x) .
$$

Application of controlled forcing (27) results in 4 independent controls, each one $v_{k}(t), w_{k}(t), v_{\ell}(t), w_{\ell}(t)$ entering one and only one of the equations (13). Under the action of (28) each of controls $v, \bar{v}$ is applied simultaneously to a pair of equations (13).

Assume $|k| \neq|\ell|, k \wedge \ell \neq 0$. We compute the bilinear form (23).

$$
\mathcal{B}(g, g)=\left(-|\ell|^{-2}+|k|^{-2}\right)\{\cos (k \cdot x), \cos (\ell \cdot x)\}
$$

Up to a scalar multiplier $\mathcal{B}(g, g)$ equals

$$
\begin{array}{r}
\left(-|\ell|^{-2}+|k|^{-2}\right) \sin (k \cdot x) \sin (\ell \cdot x)= \\
=(k \wedge \ell)\left(-|\ell|^{-2}+|k|^{-2}\right)(\cos ((k-\ell) \cdot x)-\cos ((k+\ell) \cdot x)) .
\end{array}
$$

Similarly up to a scalar multiplier $\mathcal{B}(\bar{g}, \bar{g})$ equals

$$
(k \wedge \ell)\left(-|\ell|^{-2}+|k|^{-2}\right)(\cos ((k-\ell) \cdot x)+\cos ((k+\ell) \cdot x)) ;
$$

The span of $\mathcal{B}(g, g), \mathcal{B}(\bar{g}, \bar{g})$ coincides with the span of

$$
g^{01}=\cos ((k-\ell) \cdot x), g^{21}=\cos ((k+\ell) \cdot x) .
$$

The direction

$$
\bar{g}^{01}=\sin ((k-\ell) \cdot x)
$$

is obtained from computation of $\mathcal{B}(g, \bar{g})$. 
Choose $k=(1,1), \ell=(1,0)$; then $m=k+\ell=(2,1), n=k-\ell=(0,1)$.

Computing new directions $\mathcal{B}\left(g^{01}, \bar{g}\right), \mathcal{B}\left(\bar{g}^{01}, g\right)$ we note that due to the equality $|n|=|\ell|$ they coincide with $\mathcal{B}\left(g^{01}, \sin (k \cdot x)\right), \mathcal{B}\left(\bar{g}^{01}, \cos (k \cdot x)\right)$ respectively, and their span coincide with the span of functions

$$
\left.\left.\left.\bar{g}^{12}=\sin ((k+n) \cdot x)\right), \bar{g}^{10}=\sin ((k-n) \cdot x)\right)=\sin (\ell \cdot x)\right)
$$

Similarly span of the directions

$$
\mathcal{B}\left(g^{01}, g\right), \mathcal{B}\left(\bar{g}^{01}, \bar{g}\right)
$$

coincides with the span of functions

$$
\left.g^{12}=\cos ((k+n) \cdot x)\right), g^{10}=\cos ((k-n) \cdot x)=\cos (\ell \cdot x) .
$$

Then $g-g^{10}=\cos (k \cdot x), \bar{g}-\bar{g}^{10}=\sin (k \cdot x)$.

These two functions together with the functions $g^{01}, \bar{g}^{01}$ form a quadruple, which satisfies the assumptions of the Corollary 5.2. Hence our system is accessible by means of 2 controls.

Remark 5.1. It is plausible that strong accessibility of Galerkin approximation can be achieved by one control.

5.2. Controllability of Galerkin approximations. What regards controllability, then in general bracket generating property is not sufficient for it. One has to select Lie brackets, which lead to Lie extensions, meanwhile in the previous example $\left\{g, \Delta^{-1} g\right\},\left\{\bar{g}, \Delta^{-1} \bar{g}\right\}$ a priori do not correspond to Lie extensions.

Even in the finite-dimensional case one needs a stronger result of Proposition 3.10 to prove controllability property for the Galerkin approximations. This has been accomplished in [4,27] in @D and 3D cases.

Theorem 5.3. Let $k, \ell$ satisfy (26). For any subset $\mathcal{M} \subset \mathbb{Z}^{2}$ there exists a larger set $\mathcal{M}^{\prime}$ such that Galerkin $\mathcal{M}^{\prime}$-approximation controlled by the forcing

$$
\cos (k \cdot x) v_{k}(t)+\sin (k \cdot x) w_{k}(t)+\cos (\ell \cdot x) v_{\ell}(t)+\sin (\ell \cdot x) w_{\ell}(t),
$$

is globally controllable.

The proof of this controllability result consists of iterated application of Lie extension described in the Proposition 3.10. At each step we extend the system by new controlled vector fields which according to fkl0) and (25) correspond to $f^{m \pm \ell}=\cos ((m \pm \ell) \cdot x), \bar{f}^{m \pm \ell}=\sin ((m \pm \ell) \cdot x)$.

At the end of the iterated procedure we arrive to the system with extended set of controls - one for each observed mode. This latter system evidently would be controllable.

An important case when controllability of Galerkin approximation is implied by bracket generating property regards 2D Euler equation for incompressible ideal fluid $(\nu=0)$.

Indeed in this case the drift (zero control) dynamics is Hamiltonian and it evolves on a compact energy level. By Liouville and Poincare theorems 
Poisson-stable points of this dynamics are dense and one can apply LobryBonnard theorem ([2, 19]to establish

Theorem 5.4. For $\nu=0$ the Galerkin approximation of the 2D Euler system controlled is globally controllable by means of the forcing (28).

We trust that in the case of ideal fluid the controllability of Galerkin approximation of 2D Euler system can be achieved by scalar control.

\section{Steady state Controlled directions: abstract CONTROLLABILITY RESULT FOR NS SYSTEM}

We can not apply Proposition 3.10 to infinite dimensions directly. Still the main idea of adding new controlled directions is valid for NS systems. Now we want to formulate an abstract controllability criterion, based on Lie extensions and on the computation of Lemma 4.1. This criterion will be employed in forthcoming Sections for establishing controllability of NS system on various $2 \mathrm{D}$ domains.

Theorem 6.1 (controllability of NS systen via saturation of controls). Let

$$
\operatorname{span}\left\{f^{1}, \ldots, f^{r}\right\}=\mathcal{S}=\mathcal{D}^{1}
$$

be a finite dimensional space of controlled directions. Assume $f^{1}, \ldots f^{r}$ to be steady motions of Euler equation (24). For each pair of linear subspaces $\mathcal{L}^{1}, \mathcal{L}^{2}$ consider the span of the image $\mathcal{B}\left(\mathcal{L}^{1}, \mathcal{L}^{2}\right)$ of the bilinear map (23). Define successively

$$
\mathcal{D}^{j+1}=\mathcal{D}^{j}+\operatorname{span} \mathcal{B}\left(\mathcal{S}^{j}, \mathcal{D}^{j}\right), j=1,2, \ldots
$$

where $\mathcal{S}^{j} \subseteq \mathcal{D}^{j}$ is a linear subspace spanned by steady motions. If $\bigcup_{j} \mathcal{D}^{j}$ is dense in the Sobolev space $H^{2}(M)$, then the NS system is controllable in finite-dimensional projections and $L_{2}$-approximately controllable.

As far as $\mathcal{D}^{1}$ consists of steady motions then $\mathcal{D}^{j+1} \supseteq \mathcal{D}^{j}+\operatorname{span} \mathcal{B}\left(\mathcal{D}^{1}, \mathcal{D}^{j}\right)$. Introduce the sequence of spaces

$$
\mathcal{D}_{1}^{j+1}=\mathcal{D}_{1}^{j}+\operatorname{span} \mathcal{B}\left(\mathcal{D}^{1}, \mathcal{D}_{1}^{j}\right) .
$$

Evidently $\mathcal{D}_{1}^{j} \subseteq \mathcal{D}^{j}$ and density of $\bigcup_{j} \mathcal{D}_{1}^{j}$ in $H^{2}(M)$ guarantees controllability.

Let for $f_{s} \in \mathcal{D}^{1}, D_{f_{s}}=\mathcal{B}\left(f_{s}, \cdot\right)$ :

$$
D_{f_{s}}=\left\{\Delta^{-1} \cdot f_{s}\right\}+\left\{\Delta^{-1} f_{s}, \cdot\right\} .
$$

The iterated computations (29) correspond to iterated applications of the operators $D_{f_{s}}$ to $f^{1}, \ldots f^{r}$ and taking linear span.

Corollary 6.2. Let $\mathcal{F}$ be the minimal common invariant linear subspace of the operators $D_{f_{1}}, \ldots, D_{f_{l}}$ which contains $f_{1}, \ldots, f_{k}$. If $\mathcal{F}$ is everywhere dense in $L_{2}(M)$, then the system is $L_{2}$-approximately controllable and solidly controllable in finite dimensional projections. 


\section{NAVieR-Stokes and Euler System on $\mathbb{T}^{2}$}

In this Section we formulate results regarding controllability in finitedimensional projections and $L_{2}$-approximate controllability on $\mathbb{T}^{2}$. Namely we describe sets of controlled directions which satisfy criterion provided by Theorem 6.1.

We take the basis of complex eigenfunctions $\left(e^{i k \cdot x}\right)$ of the Laplacian on $\mathbb{T}^{2}$ and take the Fourier expansion of the vorticity $w(t, x)=\sum_{k} q_{k}(t) e^{i k \cdot x}$ and control $v(t, x)=\sum_{k \in \mathcal{K}^{1}} v_{k}(t) e^{i k \cdot x}$; here $k \in \mathbb{Z}^{2}$. As far as $w$ and $f$ are real-valued, we have $\bar{q}_{n}=q_{-n}, \bar{v}_{n}=v_{-n}$. We assume $v_{0}=0$ and $q_{0}=0$.

Using the computation (25) we write the infinite system of ODE (13) as

$$
\dot{q}_{k}=\sum_{m+n=k,|m|<|n|}(m \wedge n)\left(|m|^{-2}-|n|^{-2}\right) q_{m} q_{n}-|k|^{2} q_{k}+\hat{v}_{k}(t) .
$$

The controls $v_{k}$ are nonvanishing only in the equations for the variables $q_{k}$ indexed by symmetric set $\mathcal{K}^{1} \subset \mathbb{Z}^{2} \backslash\{0\}$. For $k \notin \mathcal{K}^{1}$ the dynamics is

$$
\dot{q}_{k}=\sum_{m+n=k,|m|<|n|}(m \wedge n)\left(|m|^{-2}-|n|^{-2}\right) q_{m} q_{n}-|k|^{2} q_{k}, k \notin \mathcal{K}^{1} .
$$

There is a symmetric set of observed modes $\mathcal{K}^{o} \supset \mathcal{K}^{1}$, which we want to steer to some preassigned values. In the only interesting case where $\mathcal{K}^{1}$ is proper subset of $\mathcal{K}^{o}$, the equations indexed by $k \in \mathcal{K}^{o} \backslash \mathcal{K}^{1}$ are of the form (31). They do not contain controls and need to be controlled via state variables.

We give a hint of how this can be done; it is an infinite-dimensional version of Proposition 3.10 for NS system on $\mathbb{T}^{2}$.

Let $r, s \in \mathcal{K}^{1}, r \wedge s \neq 0,|r|<|s|$, and $k=r+s \notin \mathcal{K}^{1}$. The equations for $q_{r}, q_{s}$ contains controls $\hat{v}_{r}, \hat{v}_{s}$, while the equation for $q_{k}$ does not.

Take Lipschitzian functions $v_{r}(t), v_{s}(t),\left(v_{r}(0)=v_{s}(0)=0\right)$ and substitute $\hat{v}_{r}, \hat{v}_{s}$ in the equations for $q_{r}, q_{s}$ by $\varepsilon^{-1} \dot{v}_{r}(t)+\tilde{v}_{r}$ and $\varepsilon \dot{v}_{s}(t)+\tilde{v}_{s}$. We obtain the equations

$$
\begin{gathered}
\dot{q}_{r}=\sum_{m+n=r,|m|<|n|}(m \wedge n)\left(|m|^{-2}-|n|^{-2}\right) q_{m} q_{n}-|r|^{2} q_{r}+\varepsilon^{-1} \dot{v}_{r}(t)+\tilde{v}_{r} \\
\dot{q}_{s}=\sum_{m+n=s,|m|<|n|}(m \wedge n)\left(|m|^{-2}-|n|^{-2}\right) q_{m} q_{n}-|s|^{2} q_{s}+\varepsilon \dot{v}_{s}(t)+\tilde{v}_{s} .
\end{gathered}
$$

Introduce variables $q_{r}^{*}=q_{r}-\varepsilon^{-1} v_{r}(t), q_{s}^{*}=q_{s}-\varepsilon v_{s}(t)$. Assuming $v_{r}(T)=$ $v_{s}(T)=0$ we conclude $q_{r}(T)=q_{r}^{*}(T), q_{s}(T)=q_{s}^{*}(T)$.

Let us rewrite the infinite system of ODE (30)-(31) via $q_{r}^{*}, q_{s}^{*}$ in place of $q_{r}, q_{s}$. The right-hand side of the equation for $q_{k}=q_{r+s}$ gains the addend

$$
(r \wedge s)\left(|r|^{-2}-|s|^{-2}\right)\left(q_{r}^{*}+\varepsilon^{-1} v_{r}(t)\right)\left(q_{s}^{*}+\varepsilon v_{s}(t)\right)
$$

and we see that the controls $v_{r}, v_{s}$ enter this equation via product $v_{r}(t) v_{s}(t)$. Same $v_{r}, v_{s}$ enter this and all other equations linearly. 
Substitute $v_{j}(t)$ for $j=r, s$ by $v_{j}(t)=2^{1 / 2} \sin \left(t / \varepsilon^{2}\right) \bar{v}_{j}(t)$ with $\bar{v}_{j}(t)$ having bounded variations. Then the right-hand side of the equation for $q_{k}$ will gain the product

$$
2 \sin ^{2}\left(t / \varepsilon^{2}\right) \bar{v}_{r}(t) \bar{v}_{s}(t)=\left(1-2 \cos \left(2 t / \varepsilon^{2}\right)\right) \bar{v}_{r}(t) \bar{v}_{s}(t) .
$$

If $\varepsilon \rightarrow+0$ this product tends to $\bar{v}_{r}(t) \bar{v}_{s}(t)$ in relaxation metric. In all other equations $\bar{v}_{r}(t)$ and $\bar{v}_{s}(t)$ enter linearly and are multiplied by fast oscillating functions. Therefore the corresponding terms tend to 0 in relaxation metric.

Therefore one can pass (as $\varepsilon \rightarrow 0$ ) to a limit system which now contains 'new' control $\bar{v}_{r s}=\bar{v}_{r}(t) \bar{v}_{s}(t)$ in the equation for $q_{k}=q_{r+s}$. (This control corresponds to the control $v_{12}$ from the Proposition 3.10).

A difficult analytic part is justification of this passage to the limit. It is accomplished in [6, 7] for $\mathbb{T}^{2}$ and in [23, 24] for rectangular and other kinds of regular $2 \mathrm{D}$ domains. We refer interested readers to these publications.

Note that the new controlled direction corresponds to complex exponential which is an eigenfunction of the Laplacian on $\mathbb{T}^{2}$. Hence we can model Lie extensions and formulate controllability results in terms of indices $k \in \mathbb{Z}^{2}$ of controlled modes.

Define a sequence of sets $\mathcal{K}^{j} \subset \mathbb{Z}^{2}$ iteratively as follows:

$$
\mathcal{K}^{j}=\mathcal{K}^{j-1} \bigcup\left\{m+n \mid m, n \in \mathcal{K}^{j-1} \bigwedge\|m\| \neq\|n\| \bigwedge m \wedge n \neq 0\right\} .
$$

Definition 7.1. A finite set $\mathcal{K}^{1} \subset \mathbb{Z}^{2} \backslash\{0\}$ of forcing modes is called saturating if $\bigcup_{j=1}^{\infty} \mathcal{K}^{j}=\mathbb{Z}^{2} \backslash\{0\}$, where $\mathcal{K}^{j}$ are defined by (32).

Theorem 7.2. (controllability in finite-dimensional projection) Let $\mathcal{K}^{1}$ be a saturating set of controlled forcing modes and $\mathcal{L}$ be any finite-dimensional subspace of $H^{2}\left(\mathbb{T}^{2}\right)$. Then for any $T>0$ the $N S /$ Euler system on $\mathbb{T}^{2}$ is time-T solidly controllable in finite-dimensional projections and time-T $L_{2}$ approximately controllable.

As we see the saturating property is crucial for controllability. In [7] the following characterization of this property has been established.

Theorem 7.3. For a symmetric finite set $\mathcal{K}^{1}=\left\{m^{1}, \ldots, m^{s}\right\} \subset \mathbb{Z}^{2}$ the following properties are equivalent:

i) $\mathcal{K}^{1}$ is saturating;

ii) the greatest common divisor of the numbers $d_{i j}=m^{i} \wedge m^{j}, i, j \in$ $\{1, \ldots, s\}$ equals 1 and there exist $m^{\alpha}, m^{\beta} \in \mathcal{K}^{1}$, which are not collinear and have different lengths.

Corollary 7.4. The set $\mathcal{K}^{1}=\{(1,0),(-1,0),(1,1),(-1,-1)\} \subset \mathbb{Z}^{2}$ is saturating. Solid controllability in any finite-dimensional projection and $L_{2}$ approximately controllability can be achieved by forcing 4 modes. 


\section{Controllability of 2D NS System on a Rectangular domain}

The study of controllability in finite-dimensional projections and of $L_{2^{-}}$ approximate controllability on a rectangular domain has been accomplished by S.Rodrigues in $[23,24]$. The main idea is similar to the one employed for the periodic case, but the computations are more intricate. The reason is twofold: i) the algebraic properties of the bilinear operation calculated for the eigenfunctions of the Laplacian are more complex, and ii) one needs to care about boundary conditions.

For a velocity field $u$ on a rectangular $\mathcal{R}$ with sides of lengths $a, b(a \neq b)$ we assume Lions boundary conditions to hold. In terms of the vorticity $w$ they can be written as (11).

The (vorticity) eigenfunctions $\phi^{k}$ of the Laplacian are

$$
\phi^{k}=\sin \left(\frac{\pi}{a} k_{1} x_{1}\right) \sin \left(\frac{\pi}{b} k_{2} x_{2}\right),\left(k=\left(k_{1}, k_{2}\right) \in \mathbb{Z}^{2} .\right.
$$

To find extending controlled direction one needs to pick two eigenfunctions $f^{1}=\phi^{k}, f^{2}=\phi^{\ell}, k, \ell \in \mathbb{Z}^{2}$ and to proceed with the computation (23). This results in a linear combination of at most 4 eigenfunctions $W^{s}$.

Then again one can follow Lie extensions on two-dimensional lattice $\mathbb{Z}^{2}$ of Fourier exponents $k=\left(k_{1}, k_{2}\right)$. If the controlled modes are indexed by $k \in \mathcal{K}^{1}=\left\{\left(k_{1}, k_{2}\right) \mid 1 \leq k_{1}, k_{2} \leq 3, k \neq(3,3)\right\}$, then one can verify that after $m$ Lie extensions the set of extended controlled directions will contain all the modes $\left(k_{1}, k_{2}\right)$ with $k_{1}, k_{2} \leq m+3$ with the exception of $(m+3, m+3)$.

This leads to the following controllability result.

Theorem 8.1 (controllability on rectangular domain). Let 8 controlled $d i$ rections correspond to the functions (33) with $k \in\left\{\left(k_{1}, k_{2}\right) \mid 1 \leq k_{1}, k_{2} \leq\right.$ $3, k \neq(3,3)\}$. Then the NS system defined on the rectangular domain under Lions boundary condition is controllable in finite-dimensional projections and $L_{2}$-approximately controllable.

\section{Controllability on a generic Riemannian surface DIFFEOMORPHIC TO A DISC}

In this section we consider NS system under boundary conditions (11) on a Riemannian surface $M$. We manage to prove that for a generic surface (exact meaning of genericity will be specified in a moment) diffeomorphic to a disc one can choose 3 controlled directions, corresponding to eigenfunctions (modes) of the Laplacian on $M$, which provide controllability in finite-dimensional projections.

In what follows we assume $M$ to have $C^{\infty}$-smooth boundary and to be endowed with Riemannian metric.

The diffeomorphism $\Phi: M \mapsto D$ induces a $C^{\infty}$-smooth metrics on the disc $D$, and instead of speaking of various Riemannian surfaces we will speak of various Riemannian metrics on the fixed disc $D$. Generic Riemannian surface corresponds to a generic smooth Riemannian metric on $D$, meaning a metric 
which belongs to a residual subset of topological space of $C^{\infty}$ metrics. A subset is residual if it contains an intersection of countable family of open dense subsets of a topological space.

We will take as controlled 'directions' the modes or the eigenfunctions of the Laplace-Beltrami operator $\Delta$ corresponding to each metrics: $\Delta^{-1} f_{s}=$ $\lambda_{s}^{-1} f_{s}, \quad s=1, \ldots, l$. To apply the abstract controllability criterion formulated as Corollary 6.2 it suffices to verify that the iterated applications of $D_{f_{s}}=\left\{\Delta^{-1} \cdot, f_{s}\right\}+\left\{\Delta^{-1} f_{s}, \cdot\right\}$ to $f_{j}$ result in a dense subset of $H^{2}(M)$.

Theorem 9.1. For a generic Riemannian surface $M$ diffeomorphic to a disc there exist 3 eigenfunctions (modes) $f_{1}, f_{2}, f_{3}$ of the Laplace-Beltrami operator $\Delta$ on $M$ such that the NS system on $M$ is controllable in finitedimensional projections by means of controlled forcing applied to these modes.

Sketch of the proof. As we showed before [7] it suffices to establish controllability in projection on any finite-dimensional coordinate subspace $\mathcal{L}$ spanned by a finite number of eigenfunctions of the Laplace-Beltrami operator. According to the Corollary 6.2 we have to verify the nonnullity of some determinant, denoted $\operatorname{Det}_{\mathcal{L}}$, which is calculated via the (iterated) Poisson brackets of the functions $f_{1}, f_{2}, f_{3}$.

Assume for the moment that for some smooth metric $\mu_{0}$ on $D$ this determinant $\operatorname{Det}_{\mathcal{L}}$ is nonvanishing. Take an analytic metrics which approximates $\mu_{0}$ (analytic metrics are dense in the space of smooth metrics) and for which $\operatorname{Det}_{\mathcal{L}}$ is nonvanishing; we denote it by $\mu_{0}$ again.

Then taking any analytic Riemannian metrics $\mu_{1}$ on $D$, we construct a linear homotopy $\mu_{t}$ between $\mu_{0}$ and $\mu_{1}$ :

$$
\left.\mu_{t}\right|_{q}(\xi, \xi)==\left.(1-t) \mu_{0}\right|_{q}(\xi, \xi)+\left.t \mu_{1}\right|_{q}(\xi, \xi), 0 \leq t \leq 1 .
$$

Recall that the 'values' of Riemannian metrics at each point $q \in M$ are positive definite quadratic forms, which form a convex cone.

The dependence on $t$ of the Laplacians $\Delta(t)$ corresponding to the metrics $\mu_{t}$ is analytic.

We want to trace the evolution of a finite number of the eigenvalues $\lambda_{j}^{t}, \quad(j \in J$ - finite set $)$ and of the corresponding eigenfunctions of $\Delta(t)$ with $t$ varying in $[0,1]$. This allows us to study the restriction of $\Delta(t)$ onto a finite-dimensional space (see [20, Ch. 7]).

By classical result of perturbation theory (see [20, Ch. 2, Ch. 7]) eigenvalues $\lambda_{j}^{t}$ of an analytic family $t \mapsto A_{t}$ of linear operators are analytic with respect to $t$ beyond finite number of exceptional points in $[0,1]$. Any moment $t$ for which the eigenvalues $\lambda_{j}^{t},(j \in J)$ are pairwise distinct is nonexceptional. Singularities of the function $t \mapsto \lambda_{j}^{t}$ may occur when $\lambda_{j}^{t}$ become multiple. The eigenvectors and respective eigenprojections may have poles at the exceptional points.

The picture is much more regular for normal operators and in particular for Laplacians, which are self-adjoint. In this case the eigenvalues and the 
eigenfunctions are known to depend analytically on $t$ everywhere on $[0,1]$ ([20, Ch.2, Th.1.10]). Also the dependence of the derivatives of the eigenfunctions on $t \in[0,1]$ is analytic. Hence the determinant $\operatorname{Det}_{\mathcal{L}}$ is analytic function of $t$. As far as it is nonvanishing for $t=0$, it may vanish only at a finite number of points $t \in[0,1]$.

Take $\mu_{t}$ corresponding to all nonexceptional $t \in[0,1]$ for which $\operatorname{Det}_{\mathcal{L}}$ is nonvanishing. Among those there exist $t_{s}$ arbitrarily close to 1 . The metrics $\mu_{t_{s}}$ are arbitrarily close to $\mu_{1}$ in $C^{\infty}$-metrics; for the corresponding $\Delta\left(t_{s}\right)$ the eigenvalues of interest are distinct and $\operatorname{Det}_{\mathcal{L}}$ is nonvanishing. The dependence of the eigenfunctions and of their derivatives on metric $\mu$ is continuous in $C^{\infty}$-metric in a neighborhood of $\mu_{t_{s}}$. Hence $\operatorname{Det}_{\mathcal{L}}$ is nonzero for all $\mu$ from small $C^{\infty}$-neighborhoods of these $\mu_{t_{s}}$. Taking union of these neighborhoods we get an open set whose closure contains $\mu_{1}$. Repeating the homotopy argument for each analytic metric $\mu_{1}$ on $D$ we get an open dense in $C^{\infty}$ set of metrics for which $\operatorname{Det}_{\mathcal{L}}$ is nonvanishing.

There remains still one unsettled problem: to find a metric $\mu_{0}$ on $D$ for which the determinant $\operatorname{Det}_{\mathcal{L}}$ is nonvanishing.

This problem is by no means minor. To construct such a metric we use a result of the Remark 10.1 from the next Section. This result, obtained by S.Rordigues [26] establishes controllability of NS/Euler system on the halfsphere $\mathbb{S}_{+}^{2}$ under Navier (and in particular under Lions) boundary conditions. The metric on $\mathbb{S}_{+}^{2}$ is inherited from the embracing Euclidean space $\mathbb{R}^{3}$.

The degenerate control is applied to 3 modes - spherical harmonics which are eigenfunctions of the Laplacian on $\mathbb{S}_{+}^{2}$. This system is proved to be controllable in any finite-dimensional projection.

Mapping $\mathbb{S}_{+}^{2}$ onto the disc $D$ analytically we obtain the corresponding metric $\mu_{0}$ and the Laplacian on $D$ for which the determinant $\operatorname{Det}_{\mathcal{L}}$ is nonvanishing.

Remark 9.1. The construction of a residual set of Riemannian metrics can be transferred (almost) without alterations to the torus $\mathbb{T}^{2}$, for which we studied controllability of NS/Euler system in the Section \%. The conclusion claims that there exists a residual set of smooth Riemannian metrics on $\mathbb{T}^{2}$ such that the assumptions of the Corollary 6.2 are verified and therefore the $N S$ system is controllable in finite-dimensional projections by forcing 4 modes on $\mathbb{T}^{2}$ endowed with any of these metrics.

A pertinent question would be whether the result of the Theorem 9.1 holds for a generic sub-domain $\mathcal{Q}$ with analytic boundary in $\mathbb{R}^{2}$ endowed with Euclidean metric. By Riemann mapping theorem we can find an analytic map $\mathcal{Q} \mapsto D$ which transforms the Euclidean metric on $\mathcal{Q}$ in a metric (34) on $D$. The latter possesses zero curvature and we may think that it has a conformal form ([12, Vol. 1, §§11-13]):

$$
\mu=e^{a\left(x_{1}, x_{2}\right)}\left(d x_{1}^{2}+d x_{2}^{2}\right) .
$$


Note that the curvature of (34) equals $K=(1 / 2) e^{-a\left(x_{1}, x_{2}\right)}\left(\frac{\partial^{2} a}{\partial x_{1}^{2}}+\frac{\partial^{2} a}{\partial x_{2}^{2}}\right)$; therefore plane metrics are distinguished by the condition

$$
\frac{\partial^{2} a}{\partial x_{1}^{2}}+\frac{\partial^{2} a}{\partial x_{2}^{2}}=0
$$

On the contrary if $D$ possesses a Riemannian metric $\mu$ of form (34), which satisfies (35), then $D$ can be isometrically and analytically mapped onto a 2 D domain $\mathcal{Q}$ with Euclidean metric.

We may define the corresponding homotopy between $\mu_{0}=e^{a_{0}\left(x_{1}, x_{2}\right)}\left(d x_{1}^{2}+\right.$ $\left.d x_{2}^{2}\right)$ and $\mu_{1}=e^{a_{1}\left(x_{1}, x_{2}\right)}\left(d x_{1}^{2}+d x_{2}^{2}\right)$ as

$$
\mu_{t}=e^{(1-t) a_{0}\left(x_{1}, x_{2}\right)+t a_{1}\left(x_{1}, x_{2}\right)}\left(d x_{1}^{2}+d x_{2}^{2}\right),
$$

and advance as in the previous proof.

The only problem would be constructing a plane domain $\mathcal{Q}$ with analytic boundary and Euclidean metric for which controllability in finite-dimensional projection holds.

A good candidate could be an analytically perturbed (smoothened) rectangular $\mathcal{R}_{\varepsilon}, \varepsilon>0$. Controllability on the rectangular $\mathcal{R}$ has been established in the Section 8. We are confident that controllability holds also for $\mathcal{R}_{\varepsilon}$ with small $\varepsilon>0$, but there are still some analytic problems to be settled in the proof.

\section{NS/EULER SYSTEM ON THE SPHERE $\mathbb{S}^{2}$}

The controlled vector fields we employ in the case of $\mathbb{S}^{2}$ correspond to the eigenfunctions of the corresponding spherical Laplacian or to so called spherical harmonics. We start with their brief description.

10.1. Spherical Harmonics. In this subsection we introduce some notions and results regarding spherical harmonics; our source was mainly the book [9, Ch. 10,11] by V.I.Arnold.

Consider sphere $\mathbb{S}^{2}$ equipped with the Riemannian metrics inherited from $\mathbb{R}^{3}$ and with area 2-form $\sigma$. the latter defines symplectic structure on $\mathbb{S}^{2}$.

The eigenfunctions of the spherical Laplacian are described by the following classical result. Recall that a function $g$ is homogeneous of degree $s$ on $\mathbb{R}^{n} \backslash 0$, if $g(\kappa x)=\kappa^{s} g(x)$ for each $\kappa>0$. A function $g$ is harmonic in $\mathbb{R}^{n} \backslash 0$ if $\Delta g=0$; where $\Delta$ is the euclidean Laplacian. It is known that a harmonic homogeneous function of degree $s>0$ is extendable by continuity $(g(0)=0)$ to a harmonic function on $\mathbb{R}^{n}$. This harmonic function is smooth and therefore it must be homogeneous polynomial of integer degree $s>0$.

Theorem $10.1([9])$. Constants are eigenfunctions of spherical Laplacian (of degree 0). If a (smooth) harmonic function defined on $\mathbb{R}^{n} \backslash 0$ is homogeneous of degree $s>0$, then its restriction onto sphere is eigenfunction of the spherical Laplacian $\tilde{\Delta}$ with the eigenvalue $-s(s+n-2)$. Vice versa every eigenfunction of $\tilde{\Delta}$ is a restriction onto $\mathbb{S}^{n}$ of a homogeneous polynomial. 
Another famous result is the Maxwell's theorem ([9]), which holds in $\mathbb{R}^{3}$. It states that if $\rho(x)=\left(x_{1}^{2}+x_{2}^{2}+x_{3}^{2}\right)^{-1 / 2}$ is a fundamental solution of the Laplace equation in $\mathbb{R}^{3}$, then any spherical harmonic $a$ on $\mathbb{S}^{2}$ can be represented as iterated directional derivative of $\rho$ :

$$
a=l_{1} \circ \cdots \circ l_{n} \rho,
$$

where $l_{1}, \ldots, l_{n} \in \mathbb{R}^{3}$ and the set $\left\{l_{1}, \ldots, l_{n}\right\}$ is uniquely determined by $a$.

Our controlled directions will correspond to spherical harmonics on $S^{2}$, which are the restrictions to $\mathbb{S}^{2}$ of homogeneous functions on $\mathbb{R}^{3}$. In particular we invoke so called zonal spherical harmonics, which are iterated directional derivatives of $\rho$ with respect to a fixed direction $l$.

Let $a, b$ be smooth (not necessarily homogeneous) functions on $\mathbb{R}^{3}$; the Poisson bracket of their restrictions to $\mathbb{S}^{2}$ can be computed as follows:

$$
\left\{\left.a\right|_{S^{2}},\left.b\right|_{S^{2}}\right\}(x)=\left\langle x, \nabla_{x} a, \nabla_{x} b\right\rangle,
$$

where $\langle x, \eta, \zeta\rangle$ stays for "mixed product" in $\mathbb{R}^{3}$, calculated as the determinant of the $3 \times 3$-matrix whose columns are $x, \eta, \zeta$. From now on we omit the sign of restriction $\left.\right|_{\mathbb{S}^{2}}$ while writing Poisson bracket.

Linear functions $(l, x)$ are, of course, spherical harmonics. We denote by $\vec{l}$ the Hamiltonian field on $\mathbb{S}^{2}$ associated to the Hamiltonian $\langle l, x\rangle, x \in \mathbb{S}^{2}$. Obviously, $\vec{l}$ generates rotation of the sphere around the axis $l$. According to the aforesaid $\overrightarrow{l a}=\langle x, l, \nabla a\rangle$ is the Poisson bracket of the functions $\langle l, x\rangle$ and $a$ restricted to $\mathbb{S}^{2}$.

The group of rotations acts (by the change of variables) on the space of homogeneous harmonic polynomials of fixed degree $n$. It is well-known that this action is irreducible for any $n$ (see [9] for a sketch of the proof). In other words, the following result holds.

Proposition 10.2. Given a nonzero degree $n$ homogeneous harmonic polynomial a, the space

$$
\operatorname{span}\left\{\vec{l}_{1} \circ \cdots \circ \vec{l}_{k} a: k \geq 0\right\}
$$

coincides with the space of all degree $n$ homogeneous harmonic polynomials.

10.2. Poisson brackets of spherical harmonics and controllability. Calculating Lie extensions according to the formula (23) we obtain iterated Poisson brackets of spherical harmonic polynomials, which in general need not to be harmonic.

The following Lemma shows that there is a way of finding some harmonic polynomials among them.

Lemma 10.3. For each $n>2$ there exist a harmonic homogeneous polynomial $q$ of degree 2, and harmonic homogeneous polynomial $p$ of degree $n>2$ such that their Poisson bracket is again harmonic (and homogeneous of degree $n+1)$ polynomial. 
Proof. Let us take so called quadratic zonal harmonic function $q=\frac{\partial^{2} \rho}{\partial x_{3}^{2}}$. Being restricted to the sphere $\mathbb{S}^{2}$ this function coincides with Legendre polynomial $q\left(x_{3}\right)=3 x_{3}^{2}-1$.

Consider homogeneous harmonic polynomials of variables $x_{1}, x_{2}$. In polar coordinates they are known to have representation $r^{m} \cos m \varphi$ or alternatively as $\operatorname{Re}\left(x_{1}+i x_{2}\right)^{m}, m=1,2, \ldots$. We pick the $n$th degree polynomial $p\left(x_{1}, x_{2}\right)=\operatorname{Re}\left(x_{1}+i x_{2}\right)^{n}$.

According to (36) the Poisson bracket of $q, p$ equals

$$
\{q, p\}=\left\langle x, \nabla q, \nabla p_{n}\right\rangle=\left|\begin{array}{ccc}
x_{1} & 0 & p_{x_{1}}^{\prime} \\
x_{2} & 0 & p_{x_{2}}^{\prime} \\
x_{3} & 6 x_{3} & 0
\end{array}\right|=-6 x_{3}\left|\begin{array}{ccc}
x_{1} & 0 & p_{x_{1}}^{\prime} \\
x_{2} & 0 & p_{x_{2}}^{\prime} \\
x_{3} & 1 & 0
\end{array}\right| .
$$

By (36) the latter determinant coincides with $\vec{e}_{3} p\left(x_{1}, x_{2}\right)$, where $e_{3}=$ $(0,0,1)$ is the standard basis vector of $\mathbb{R}^{3}$. Hence by Proposition 10.2 the value of this determinant is a harmonic polynomial of degree $n$; it equals $\tilde{p}\left(x_{1}, x_{2}\right)=-x_{1} p_{x_{2}}^{\prime}+x_{2} p_{x_{1}}^{\prime}$ and therefore does not depend on $x_{3}$.

Then $\{q, p\}=-6 x_{3} \tilde{p}\left(x_{1}, x_{2}\right)$. Since both $-6 x_{3}$ and $\tilde{p}$ are harmonic, we get $\Delta\{q, p\}=2 \nabla\left(-6 x_{3}\right) \cdot \nabla \tilde{p}=-12 \partial \tilde{p} / \partial x_{3}=0$.

Theorem 10.4. Consider NS/Euler system on sphere $\mathbb{S}^{2}$. Let (constant) controlled vector fields correspond to three independent linear spherical harmonics $l^{1}, l^{2}, l^{3}$, one quadratic harmonic $q$ and one cubic harmonic $c$. Then this set of controlled vector fields is saturating and the NS/Euler system is controllable in finite-dimensional projections.

Proof. It suffices to verify the assumption of the Corollary 6.2. Without lack of generality we may think that $q=\tilde{q}$ - the second degree zonal harmonic from the previous lemma. Indeed otherwise we may transform $q$ into $\tilde{q}$ by taking iterated Poisson brackets with the linear harmonics $l^{1}, l^{2}, l^{3}$.

In fact taking iterated Poisson brackets of $q$ and $c$ respectively, with $l^{1}, l^{2}, l^{3}$ we obtain all quadratic and cubic harmonics. Thus we manage to obtain all the harmonics of degrees $\leq 3$.

Let us proceed by induction with respect to the degree of harmonics. Assume that all harmonics of degrees $\leq n$ are already obtained by taking iterated Poisson brackets of $\left\{l^{1}, l^{2}, l^{3}, q, s\right\}$. Pick the harmonic polynomial $p$ constructed in Lemma 10.3; its Poisson bracket with $q$ is homogeneous harmonic polynomial $\bar{p}$ of degree $n+1$. Taking iterated Poisson brackets of $\bar{p}$ with $l^{1}, l^{2}, l^{3}$ we obtain all polynomials of degree $n+1$.

Remark 10.1. Following the lines of the previous proof S.Rodrigues established [26] controllability of NS/Euler system on the half-sphere $\mathbb{S}_{+}^{2}$. One can force 3 modes - spherical harmonics on $\mathbb{S}_{+}^{2}$ - in order to guarantee controllability in finite-dimensional projections. The details will appear elsewhere.

Remark 10.2. Applying the argument similar to the one involved in the previous Section one can conclude that there exists a residual set of smooth 
Riemannian metrics on $\mathbb{S}^{2}$ such that the assumptions of the Corollary 6.2 are verified and therefore the NS system is controllable in finite-dimensional projections by forcing 5 modes on $\mathbb{S}^{2}$ endowed with any of these metrics.

\section{REFERENCES}

[1] Agrachev A.A., R.V.Gamkrelidze, Exponential representation of flows and chronological calculus, Math. USSR Sbornik, 35(1979), 727-785.

[2] Agrachev A.A., Yu.L.Sachkov, Lectures on Geometric Control Theory, SpringerVerlag, 2004.

[3] Agrachev A.A., A.V.Sarychev, On reduction of smooth system linear in control, Math. USSR Sbornik, Vol.58, 1987, pp.15-30.

[4] Agrachev A.A., A.V.Sarychev, Navier-Stokes Equation Controlled by Degenerate Forcing: Controllability of Finite-Dimensional Approximations, In Proc. of Intern. Conf. 'Physics and Control 2003, S.Petersburg, Russia, August 20-22, 2003, CD ROM, 1346-1351.

[5] Agrachev A.A., A.V.Sarychev, Controllability of the Navier-Stokes Equation by Few Low Modes Forcing, Doklady of Russian Academy of Sciences, v.394, N.6, 2004. English translation in: Doklady Mathematical Sciences, v. 69, N.1/2,2004,pp.112-115.

[6] Agrachev A.A., A.V.Sarychev, Navier-Stokes Equations: Controllability by Means of Low Modes Forcing, J. of Mathematical Fluid Mechanics, 7(2005), pp.108-152.

[7] Agrachev A.A., A.V.Sarychev, Controllability of 2D Euler and Navier-Stokes Equations by Degenerate Forcing, Communications in Mathematical Physics, vol. 265 (2006), 673-697.

[8] Arnold V.I. Mathematical methods of classical mechanics. Springer-Verlag, 1997.

[9] Arnold V.I. Lectures on Partial Differential Equations. Springer-Verlag, 2004.

[10] Arnold V.I., Khesin B.M. Topological Methods in Hydrodynamics, SpringerVerlag, New York, 1998.

[11] Coron J.-M., Return method: some applications to flow control, In "Mathematical Control Theory", ICTP Lecture Notes Series Volume VIII-Parts 1 \& 2, 2002.

[12] Dubrovin B., S.Novikov, A.Fomenko, Modern Geometry - Methods and Applications. Part I. Springer-Verlag, New York, 1984.

[13] E W., J.C. Mattingly, Ergodicity for the Navier-Stokes Equation with Degenerate Random Forcing: Finite Dimensional approximation, Comm. Pure Appl. Math., 54 (2001), no. 11, 1386-1402.

[14] Fursikov A.V., Optimal Control of Distributed Systems. Theory and Applications, AMS, Providence, 2000.

[15] Fursikov A.V., O.Yu. Imanuilov, Exact controllability of the Navier-Stokes and Boussinesq equations, Russian Mathematical Surveys, 54(1999), Number 3,565618.

[16] Gamkrelidze R.V., On some extremal problems in the theory of differential equations with applications to the theory of optimal control, J. Soc. Ind. Appl. Math., Ser. A: Control, 3(1965) 106-128.

[17] Gamkrelidze R.V., Principles of Optimal Control Theory, Plenum Press,New York, 1978.

[18] Hairer M., Mattingly J.C., Ergodicity of the 2D Navier-Stokes Equations with Degenerate Stochastic Forcing, Annals of Mathematics, 164, no. 3, 2006.

[19] Jurdjevic V., Geometric Control Theory, Cambridge University Press, 1997.

[20] Kato T., Perturbation theory for linear operators. Springer-Verlag, 1966. 
[21] Ladyzhenskaya O.A. The mathematical theory of viscous incompressible fluid. Gordon and Breach, 1969.

[22] McShane E.J. Generalized curves, Duke Math. J. 6(1940), 513-536.

[23] Rodrigues S. Navier-Stokes Equation on the Rectangle: Controllability by Means of Low Mode Forcing, J. Dynamical Control Systems, 12(2006),517-562.

[24] Rodrigues S. Navier-Stokes Equation on a Plane Bounded Domain: Continuity Properties for Controllability. Proc. CTS Workshop.

[25] Rodrigues S., Controlled PDE on Compact Riemannian Manifolds: Controllability Issues, submitted to Proc. Workshop Mathematical Control Theory and Finance, Lisbon, Portugal, 2007.

[26] Rordigues S., A Saturating Set on Half-Sphere, private communication.

[27] Romito M., Ergodicity of finite-dimensional approximations of the $3 D$ Navier-Stokes equations forced by a degenerate noise, J. Statistical Physics, 114(2004),155-177.

[28] Shirikyan A., Approximate Controllability of Three-Dimensional Navier-Stokes Equations, Commun. Mathem. Physics, 266(2006), 123-151.

[29] Shirikyan A., Exact controllability in projections for three-dimensional NavierStokes equations, Annales de l'IHP, Analyse Non Linéaire, in press, available on-line.

[30] Young, L. C. Generalized curves and the existence of an attained absolute minimum in the calculus of variations, Comptes Rendus de la Société des Sciences et des Lettres de Varsovie, classe III, 30 (1937), 212-234.

${ }^{1}$ International School for Advanced Studies (SiSSA), Trieste, Italy \& V.A.Steklov Mathematical Institute, Moscow, Russia

2 DiMad, University of Florence, Italy

E-mail address: agrachev@sissa.it, asarychev@unifi.it 